\title{
ENTRE LO FLEXIBLE Y LO COMPLEJO: TIEMPO Y PERCEPCIÓN EN ENRIQUE X. MACÍAS (1958-1995)*
}

Data recepción: 2013/12/20

Data aceptación: 2014/07/09

Contacto autor: taboada@fyl.uva.es
Carlos Villar-Taboada

Universidad de Valladolid

\section{RESUMEN}

Enrique X. Macías (1958-1995) fue autor de una música fuertemente condicionada por su afán de componer con rigor intelectual. Desarrolló una carrera de notable proyección internacional gracias a su utilización de principios estructuradores que combinaban la flexibilidad y la complejidad y manifestando una consideración reflexiva del tiempo y la percepción en la música. Para demostrarlo, completo un selectivo examen de su producción a través del comentario analítico de diferentes composiciones que abarcan toda su trayectoria. El texto consta de una puesta al día del estado de la cuestión, un breve retrato biográfico, una aproximación analítica musical con algunos ejemplos representativos de las técnicas más habituales y unas conclusiones sobre las consecuencias estéticas de su catálogo de obras.

Palabras clave: Enrique X. Macías, música contemporánea, Galicia (España), análisis musical, tiempo musical

\section{ABSTRACT}

An author of music strongly conditioned by his eagerness to compose with intellectual rigour, Enrique X. Macías (1958-1995) enjoyed considerable international renown thanks to his use of structural principles combining flexibility and complexity, while also taking a considered approach to time and perception in music. In demonstrating this, I have undertaken a very selective examination of his work, providing an analytical commentary of various compositions encompassing his entire career. This text comprises an update of the study of his work, a brief biography, an analytical overview of his music with some representative examples of the techniques he most commonly used, and some conclusions on the aesthetic influence of his catalogue of works.

Keywords: Enrique X. Macías (1958-1995), contemporary music, Galicia (Spain), musical analysis, musical time

\section{Introducción: hacia un estado de la cuestión}

Una de las figuras más relevantes, y no exenta de cierta polémica, en el panorama de la música gallega contemporánea, ${ }^{1}$ es Enrique Xerardo Macías Alonso (Vigo, 1958-1995). Coetáneo de Manuel Balboa (1958-2004), desarroIló, como el coruñés, una destacada trayectoria entre los compositores españoles de su generación, trágicamente truncada por un prematuro fallecimiento.

La producción de este músico viajero ha sido objeto de investigaciones que abren las puertas a futuras monografías necesariamente atentas a las cuestiones más relevantes de su lenguaje y de su estética. Seguidor de una postura musical excepcional en el panorama gallego, que podría circunscribirse entre las tendencias estructuralistas postseriales, fue víctima tanto de críticas severas como de sobrevaloraciones igualmente excesivas; en cualquier caso, su música no ha producido indiferencia. De ello da buena prueba la publicación de prácticamente todas sus partituras, algunas de las primeras por la Fundación Juan March, la Editorial de Música Española Contemporánea y la francesa Jobert, y la mayoría -entendiendo por tal el conjunto de obras finalmente reconocido como integrantes del catálogo- a cargo de la portuguesa Miso Music, liderada por el también compositor Miguel Azguime (Lisboa, 1960), depositaria de los 
derechos de distribución y editora que también ha preparado un total de cinco producciones discográficas monográficamente dedicadas al compositor, que completan los registros sonoros efectuados por diversas radios europeas, así como los recogidos en discos de vinilo. ${ }^{2}$ Muchos de estos materiales son accesibles en el Archivo de Compositores Contemporáneos custodiado en la Biblioteca Española de Música y Teatro Contemporáneos de la Fundación Juan March, en Madrid. Conviene precisar que finalmente, y gracias a un convenio con la Xunta de Galicia, Miso Music ofrece acceso electrónico en línea a las partituras, lo que facilita al máximo la consulta de estas obras por parte de intérpretes e investigadores. ${ }^{3}$

Respecto a la bibliografía, resulta relativamente abundante. Hay publicadas varias entrevistas con el compositor, de extensión y profundidad variables, entre las que destaca la de García Alcalde, que ofrece un eficaz retrato de su visión de la música. ${ }^{4}$ La etapa inicial del compositor fue atendida por Soutelo -con quien estaba muy vinculado en sus primeros tiempos- desde la óptica de la agrupación artístico-musical Letrina Musicæ ${ }^{5}$-, mientras que Carreira relató las orientaciones de algunas de sus composiciones iniciales, que finalmente fueron descatalogadas. ${ }^{6}$ El relieve de su figura queda atestiguado por su presencia en diversas publicaciones importantes, a cargo de Marco, Cabañas Alamán, Rodeiro y Villanueva, ${ }^{7}$ incluyendo un volumen monográfico en la colección "Catálogos de compositores españoles de la SGAE" ${ }^{8}$ y un artículo en el diccionario español de referencia. ${ }^{9}$

Se constatan discrepancias sobre su catálogo, derivadas del afán del compositor por revisar continuamente sus obras -reaprovechando e incluso destruyendo materiales en no pocas ocasiones-, así como de la imposibilidad de reunir todo el legado en un único archivo tras su fallecimiento. Estas circunstancias condicionan que la definición actual del catálogo consista en el expuesto en la web del Centro de Informação da Música Portuguesa, basándose en la documentación de Miso Music. ${ }^{10}$ El estado de casi constante provisionalidad de las obras -y, consecuentemente, del catálogo-, puede ser valorado como una consecuencia lógica de los plantea- mientos estéticos y técnicos del autor, quien venía a considerar que en música son enormes las posibilidades para elaborar los materiales iniciales, de modo que la obra difícilmente finaliza por agotamiento del gesto generativo del comienzo, sino que concluye porque el autor pone fin voluntariamente a su «potencial de actualización». Por otra parte, esta actitud no es ni mucho menos infrecuente entre los compositores del siglo $x x, y$ hallamos el fenómeno repetido incluso en personalidades de la talla del francés Pierre Boulez (1926). Por último, más recientemente, se ha iniciado una indagación analítica ${ }^{11}$ de la producción creativa del músico donde, dado el tipo de lenguaje armónico que habitualmente empleaba, se ha aplicado una metodología derivada de los ensayos clásicos de Forte sobre la teoría de conjuntos de clases de notas. ${ }^{12}$

El presente artículo prosigue lo iniciado en la historiografía precedente, actualizando y ampliando lo allí aportado sobre dos aspectos que juzgo destacados: el tiempo y la percepción en el pensamiento compositivo de Macías.

Las búsquedas creativas que el examen de sus partituras pone al descubierto delatan una singular atracción por las investigaciones sobre la estructuración del discurso musical, tema sobre el que especuló, debatiéndose entre la flexibilidad microformal y una concepción global caracterizada por el diseño de relaciones complejas en diversos niveles de articulación discursiva. Las reflexiones acerca de los mecanismos que actúan sobre la percepción del tiempo musical y el papel de la memoria en estos procesos están presentes, como telón de fondo, a lo largo de todo su catálogo. Con estas ideas como referentes, propongo a continuación un acercamiento a la aventura musical de Enrique X. Macías.

\section{Breve aproximación biográfica}

La breve pero intensa carrera compositiva de Macías se encuentra bien surtida de importantes reconocimientos en forma de premios y encargos, que dan una buena medida de su proyección internacional. Entre los galardones se destacan: Premio del Tribuna Internacional Gaudeamus, de Holanda (1981, por Polifonías 1), Premio de Composición Cristóbal Halffter 
(1983, por Afónica), Premio de la Tribuna de Jóvenes Compositores de la Fundación Juan March (1983 y 1987, por Morgengesang // y Souvenir $n^{\circ} 1$, respectivamente), Primer Premio y accésit del Concurso de Composición de la Orquesta Nacional de España (1984, por Nachtmusik III y Letztes Urlicht, respectivamente), Mención de Honor en el Concurso Internacional Fernando Pessoa (1985, por Lisbon Revisited), Accésit del Concurso de Composición de la Joven Orquesta Nacional de España (1986, por Morgengesang III) y Premio de Composición de la SGAE (1987, por Tránsito).

Igualmente relevante es la relación de encargos recibidos: Radio Finlandesa (1980 Viagem de Inverno), Fundação Calouste Gulbekian de Lisboa (1981, Morgengesang I), Centro de Bellas Artes de Madrid (1985, Langsam), Ministerio de Cultura (1985, El itinerario de las palmeras), INAV GRM de Paris (1986, La lyre du désert y 1988, Portrait du matin), Unión Europea de Radiodifusión (1986, Morgengesang III), Tage für neue Musik (1988, Nobilissima Visione II), Centro para la Difusión de la Música Contemporánea de Madrid (1989, Nobilissima Visione II/Postludios ; y 1992, La chambre dans l'espace), XII Festival Internazionale Antidogma Musica de Turín (1989, Clare I y Extracto), Radio France (Duplo), Concello de Vigo (1992, Exequias), Xunta de Galicia para Galicia no tempo (1993, Iubilaeum), INAEM (1994, Alias), Centro Galego de Arte Contemporánea (1995, Itinerario de luz), Festival Música Viva de Parede, en Portugal (1995, Antistrofas) y Festival de Música de Canarias (1995, Clamores e alegorías).

Parece haber desempeñado un papel desencadenante de su vena creativa el temprano contacto en Vigo con Rudesindo Soutelo, quien lo acompañó en las actuaciones del grupo «Letrinae Musica» le facilitó sus primeros estrenos en el marco del I Encuentro de Artistas Jóvenes, en el contexto de una ciudad protagonista de la «movida» y culturalmente destacada, con agrupaciones como el Grupo de Comunicación Poética «Rompente». En los años inmediatamente posteriores participó de manera activa en la organización del «Festival de Vran» de la misma ciudad -continuador, en cuanto a espíritu, de la iniciativa precedente-, y se enroló, para labores de crítica musical, en las páginas viguesas de $L a$ Voz de Galicia y Pueblo Gallego. Parece bastante probable que la experiencia en cuanto a gestión y organización derivada del «Festival de Vran» le resultase satisfactoria, ya que años después se vio implicado en tareas similares con motivo de las «Xornadas de Música Contemporánea» de la universidad compostelana, a partir de 1987, en colaboración con el profesor Carlos Villanueva Abelairas.

Su formación musical fue netamente autodidacta, con unos inicios al margen de los convencionalismos de los conservatorios, pero buscó mediante becas, en muchos casos- la asistencia a cursos especializados en la creación musical contemporánea para conocer, desde sus protagonistas, las últimas tendencias y las técnicas emergentes en la composición musical. Parece probable que esta decisión fuese tomada considerando críticas dirigidas a sus primeras creaciones, datadas en los setenta y pronto retiradas del catálogo, donde se acusaban una falta de estructuración y unas carencias técnicas que no son sino fácilmente comprensibles en cualquier músico rondando los veinte años.

Así se comprende su asistencia a los Encontros de Música Contemporánea de la Fundación Gulbekian de Lisboa (1979), a la Internationale «Gaudeamus» Musieckweek de Amsterdam y a los Ferienkürse de Darmstadt, que continuó visitando en años posteriores, hasta 1984, gracias a nuevas becas del Institut für die Neue Musik de la ciudad alemana, así como sus estancias en centros de prestigio como los estudios de música electrónica de la Radio Finlandesa (1981) y de la Academia de Música de Cracovia, en Polonia (1982) y el Gabinete de Música Electroacústica de Cuenca (1984), o su presencia en calidad de compositor invitado en el Instituto de Sonología de Utrecht y en el Studio for Electro-Instrumental Music de Amsterdam, ambos en Holanda (1986 y 1988), o su participación en el Encuentro de Composición de Villafranca del Bierzo, con Cristóbal Halffter (1985), el Espace Musical de París (1987 y 1988) y en el stage de composición del Institut de Recherche et Coordination Acoustique/Musique (IRCAM, París, 1992). Como consecuencia de todos estos viajes, Macías estuvo en condiciones de conocer, de un modo muy 
directo, las modas estilísticas vigentes en el panorama europeo de la música contemporánea. $Y$, a juzgar por los premios cosechados y por la entidad de los encargos que disfrutó, supo aprovechar intensamente las oportunidades que brindaban esos foros. No se puede olvidar que este compositor pretendió -y, en buena medida, logró- vivir de su música.

Protagonista de una carrera rotundamente fulgurante, cuajada de abundantes reconocimientos, fue un compositor muy viajero y uno de los que gozó de una más amplia proyección tanto en su país como, especialmente -y esto merece destacarse-, en el extranjero.

La irrupción de Macías en la escena compositiva gallega de finales de los setenta supuso una ruptura radical con las poéticas anteriores, ligadas en mayor o menor medida al mundo tonal. La excelente acogida de su producción, como se constata mediante los premios y los encargos, lo erigió como un símbolo de la creación musical «vanguardista» y de la renovación cultural gallega.

Tras su fallecimiento, se promovieron diversas iniciativas concebidas para preservar su memoria manteniendo viva su música. Nacieron así los ciclos de conciertos que, bajo la denominación de «Memorial Enrique X. Macías» se celebraron en Vigo de 2001 a 2003, bajo la dirección artística de Manuel Rodeiro y el apoyo institucional del concello vigués. Más recientemente, en el proyecto "As nosas músicas, os nosos sons", de la Dirección Xeral de Creación e Difusión Cultural de la Xunta de Galicia, en 2008, se homenajeó el quincuagésimo aniversario del nacimiento de Macías -en una iniciativa que también se dedicó a Balboa-.

\section{Estructura y tiempo musical en la música de Enrique $X$. Macías}

En 1979, Carreira cita un catálogo integrado ya por veinte composiciones, de las que Cabañas Alamán apenas reflejará Siete micropiezas para piano, Poema de Requiem y Polifonías ${ }^{13}$. Se observa la búsqueda de una progresiva ampliación de medios, constatándose aproximaciones a la orquesta (Alternancias y Fracturas) y, sobre todo, a la cinta magnética y la electrónica (Secuencias,
Diálogos monologados, Poema de Requiem, Longa noite de pedra, Cariátides), ámbito del que parece haber sido un auténtico pionero en Galicia.

Años más tarde, el compositor Tomás Marco señaló a Macías como uno de los «Jóvenes maestros», al lado de figuras de talla indiscutible en la actualidad como Francisco Guerrero, José Ramón Encinar o José Luis Turina, entre otros. Este juicio es bastante más sustancial que la posible adscripción al -hipotético- «Grupo del Bierzo», formado en torno a los cursos de composición de Cristóbal Halffter en Villafranca, con que también se le ha relacionado. Destaca Marco una capacidad expresiva que parece redundar en un exceso de complicación formal: ${ }^{14}$

Macías posee talento y fino sentido poético, aunque muchas de sus obras tienen una enorme complejidad de realización y, a veces, la escritura es más compleja de lo que correspondería a la ideación musical [...].

La obra de Macías es atractiva y estimulante porque tiene el sabor de lo experimental $y$, al propio tiempo, un sentido poético. Importante ya en su generación, alcanzará sin duda cotas muy altas en cuanto pueda precisar el lenguaje que necesita para su ideación.

Por su ya comentada proximidad al compositor, el juicio del profesor Villanueva adquiere un interés notorio, con un doble valor documental y crítico. Entre otras aportaciones, cabe mencionarse su estimulante -por humana- semblanza personal, cuando lo califica como «buen estructuralista» y recuerda su carácter "escrupuloso, detallista, meticuloso no proceso creador», ${ }^{15} \mathrm{O}$ cuando, retratando el acercamiento epistolar entre el vigués y Jesús Bal y Gay (1905-1993), «de espaldas a las tendencias de la sociedad gallega en la que quisieron vivir», subraya su compromiso «contra la línea más tradicionalista, pasadista, "enxebrista" y hasta populista». ${ }^{16}$ Ciertamente, la actitud así descrita es identificable desde las primeras creaciones.

Siete micropiezas (1978) $)^{17}$ puede considerarse un modelo de la primera etapa, que sigue el estilo aforístico del Anton Webern de Sechs Bagatellen, op. 9 (1913), con las que desde luego comparte la brevedad y el uso de una armo- 
nía atonal, organizada con una gran libertad de planteamientos, y el uso de elementos repetitivos en forma de pequeños ostinati. Además, se suscitan juegos semánticos gracias a la introducción de citas de composiciones previas (relacionadas con la música popular gallega). El carácter miniaturesco de estas breves piezas y el tratamiento motívico de que son objeto alejan su arquitectura formal de los modelos tradicionales, articulándose en función de las relaciones que se establecen entre sonoridades y texturas diferentes, calculadas de manera intuitiva pero con eficacia suficiente como para lograr en cada micropieza una coherencia estructural clara.

Foglio I (1981-1982), ${ }^{18}$ para clave, presenta una escritura instrumental ágil y flexible, dinamizada por la ausencia de barras de compás. Al inicio de la obra se observan tres motivos generadores, fácilmente combinables entre sí. Primero, el estatismo máximo que representa un pedal en do, que será continuo a lo largo de toda la composición (motivo 0). Toda expansión de los demás materiales actuará como una especie de adorno en relación con esta sonoridad básica, tratada como un auténtico polo de atracción al que tiende la música. Una segunda idea (motivo 1) consiste en un heptacordo equilibrado rítmica y armónicamente. La última idea (motivo 2) consiste en un gran conglomerado de notas que sólo carece de fa y fa\#, que se va transformando mientras preserva su identidad gracias a la repetición de dos tricordos, usados como invariancias -como elementos que permanecen inalterados durante las sucesivas transformaciones- para garantizar la coherencia: (sol-)do-si-do\# y sol-lasol\#(-do).

En esta composición el orden de los elementos desempeña un papel muy importante como mantenedor de la identidad -y de la continuidad- en el proceso de perpetua transformación motívica. Sólo al principio se producen repeticiones casi literales de los motivos 1 y 2, ya que en cada una de las sucesivas apariciones se presentan con alguna novedad, como alteraciones en el orden de los elementos, combinaciones entre motivos, diferencias en las sucesiones interválicas, transportes parciales que no afectan a todo un motivo, inserciones de nuevos elementos o alteraciones del diseño rítmico precedente. Lo más curioso es que todos los recursos transformativos parecen aplicarse a unos materiales básicos que asumen una doble función de origen y destino, en especial la nota eje do. El análisis evidencia una lógica clara en todo el proceso: una perenne transformación, que revisita y transforma de manera continua los tres motivos generadores. La lógica queda patente, incluso, en el funcionamiento de una incipiente sintaxis, identificable en la asignación de funciones estructurales a determinados elementos (las repeticiones de do\# señalan un fin de ciclo, el motivo 1 aparece antes que el motivo 2, los hitos en los registros seccionan el discurso, etc.). El discurso se ordena sobre todo en torno al motivo 0 , que regula las barras verticales (no de compás) separadoras de las secciones en la partitura:

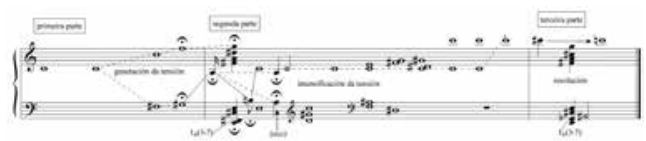

Ejemplo 1: Evolución del motivo 0 en Foglio I (1981-1982)

Se observa entonces una seccionalización tripartita: un inicio que salta de lo estable hacia la tensión, una parte central que es la más inestable y una parte final que retorna lentamente hacia el comienzo. La música describe un proceso que, impelido por las transformaciones, termina regresando, en la enésima mutación, a su origen.

Souvenir $n^{\circ} 1$ pour neuf instruments (19811982) es una pieza mayor que las precedentes. La plantilla consta de dos pianos, un trío de maderas (flauta, oboe y clarinete), un glockenspiel y un trío de cuerdas (violín, viola y violonchelo). Esta infrecuente distribución sugiere un interés por la espacialización tímbrica, con el glockenspiel en el centro de la escena y, a cada lado, un piano y un trío. Los pianos y el glockenspiel unifican la tímbrica y el espacio sonoro mediante su capacidad para la reverberación; mientras, los tríos actúan como dos fuerzas contrapuestas.

A diferencia de lo que parece ser más típico en las obras de este compositor, Souvenir $n^{\circ}$ 1 discurre de modo continuo y su partitura no presenta secciones. La escritura muestra gestos característicos del postestructuralismo más 
directamente derivado de la línea weberniana: abundancia de saltos de registro, variedad de ataques, gran libertad rítmica con frecuentes cambios de compás y de metro e inserción de grupos irregulares, uso intenso del silencio con fines expresivos y amplio rango dinámico. Pero la concepción constructiva, relacionada con la de Foglio I, consiste en una especie de juego combinatorio generado desde una colección de alturas muy concreta, identificadas con un registro en particular. Abarcan casi cinco octavas, usan el registro como parámetro diferenciador, se distribuyen a partir de la nota eje reb-do\# y contienen el total cromático:

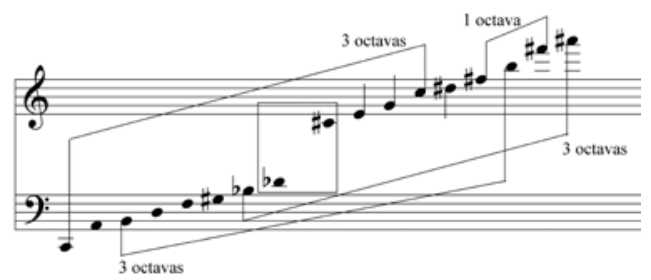

Ejemplo 2: Colección inicial de alturas para Souvenir $n^{\circ} 1$ (1981-1982)

El discurso se genera mediante la combinación de estos elementos en motivos susceptibles de ser desintegrados y recombinados, manteniendo siempre la asociación única entre cada nota y su registro. En algunos pasajes, Macías hace uso de conceptos combinatorios simples, como las permutaciones que analizo a continuación:

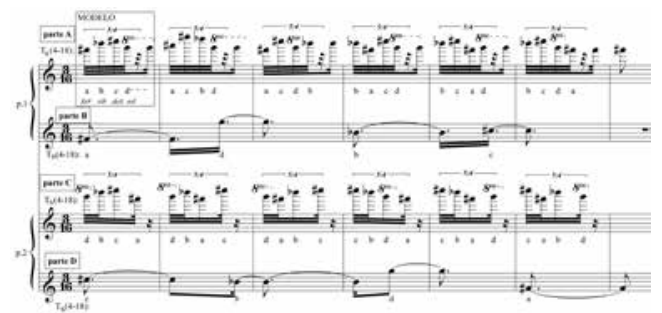

Ejemplo 3: Análisis de un fragmento combinatorio (cc. 29-35) de Souvenir $n^{\circ} 1$ (1981-1982)

Ello permite lograr un efecto de saturación armónica, como se reproduce en el ejemplo 3 (aquí un constante tetracordo 4-18), compensado mediante una superficie sonora en constante movimiento gracias a la aplicación de una combinatoria básica (permutaciones) aplicada al ritmo y al orden de las alturas del tetracordo. Macías permuta estos elementos repitiendo la secuencia de ordenación de los compases 29-31 en los 32-34, y utilizando una lógica aplastante en el plano microestructural:

\section{Patrones rítmicos básicos (compás de 3/16, $1=$ semicorchea, tachado= silencio):}

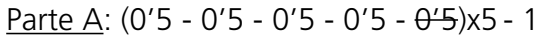

Parte B: 4'5 - 4'5 - 4'5 - 4'5

Parte C: $\left(0^{\prime} 5-0^{\prime} 5-1-\quad-0^{\prime} 5\right) \times 5-7$

Parte D: 5 - 5 - 5 - 5

Patrones combinatorios. Relación entre las permutaciones armónicas de $\mathrm{T}_{6}$ (4-18):

[en letras: orden de clases de notas; en números: orden de elementos, siempre en función del modelo]

$\begin{array}{lrr}\text { Parte A } & \text { (cc. 29-31) } & \text { (cc. 32-34) } \\ \text { abcd } & {\left[A_{1}\right] 1234} & \text { bacd }\left[A_{4}\right] 1234 \\ \text { acbd } & {\left[A_{2}\right] 1324} & \text { bcad }\left[A_{5}\right] 1324 \\ \text { acdb } & {\left[A_{3}\right] 1342} & \text { bcda }\left[A_{6}\right] 1342\end{array}$

$\underline{\text { Parte B: }}$

adbc [B] 1234

Parte C: (cc. 29-31)

(cc. 32-34)

dbca $\quad\left[C_{1}\right] 1234$

dabc $\quad\left[C_{3}\right] 1423$

cbda $\left[C_{4}\right] 1234$

cabd $\left[\mathrm{C}_{6}\right] 1423$

Parte D:

cbda [D] 1234

dbac $\quad\left[C_{2}\right] 1243 \quad$ cbad $\left[C_{5}\right] 1243$

Similitudes combinatorias:

$$
\begin{gathered}
A_{1} \equiv A_{4} \equiv C_{1} \equiv C_{4} \equiv B \equiv D \\
A_{2} \equiv A_{5}, C_{2} \equiv C_{5}, A_{3} \equiv A_{6}, C_{3} \equiv C_{6} \\
C_{4}=D=R(B)
\end{gathered}
$$

[= significa identidad (afecta a elementos y orden); $\equiv$ significa equivalencia (sólo afecta al orden); R es retrogradación] Cuadro 1: Patrones combinatorios en un fragmento (cc. 2935) de Souvenir $n^{\circ} 1$ (1981-1982)

Para examinar la lógica constructiva macroformal tomaré como muestra Souvenir $n^{\circ} 2$ (1983), en su versión A (sin electroacústica), para sexteto (flauta, clarinete bajo, vibráfono, celesta, guitarra y violonchelo). La conexión con Souvenir $n^{\circ} 1$ no reside tanto en una tímbrica proclive a la reverberación como en el empleo de tácticas combinatorias. Es más: Macías intenta en Sou- 
venir $n^{\circ} 2$ extrapolar al ámbito macroestructural los juegos combinatorios que explicaban la construcción microestructural en Souvenir $n^{\circ}$ 1. Así, ahora el compositor aplica procedimientos parecidos para distribuir la dinámica, las células rítmicas, la velocidad metronómica (siempre indicada para la negra) y los instrumentos escogidos para caracterizar las diversas secciones.

El ritmo es utilizado como germen de la unidad discursiva planificándose mediante cuatro módulos rítmicos básicos (cuatrillo de fusas, tresillo de semicorcheas, semicorcheas normales y semicorcheas de quintillo) que se combinan entre sí para derivar nuevos patrones rítmicos. Se trata de una nueva muestra de cómo Macías entiende la unidad en lo múltiplo:

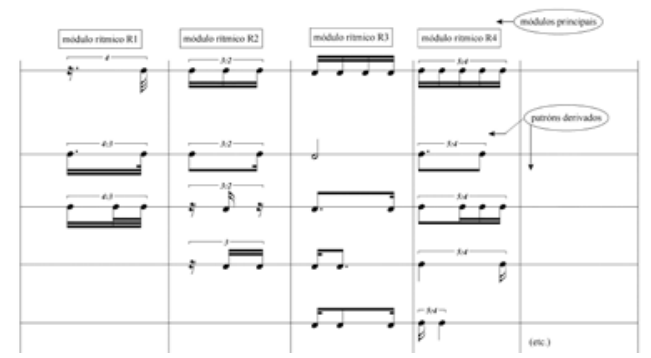

Ejemplo 4: Módulos rítmicos principales y patrones rítmicos derivados en Souvenir $n^{\circ} 2$ (1983)

De fóra (1985), para doble cuarteto solista y doble coro (con los cuatro grupos integrados por soprano, contralto, tenor y bajo) es una interesante incursión en el ámbito vocal, donde también se comprenden $O$ sol sobre os teus braços (1984), para doble cuarteto vocal, y Lisbon revisited (1984-1985), para dieciséis voces y orquesta. El compositor exige una disposición particular de los cuatro grupos vocales (los dos coros y los dos cuartetos solistas) que parece expresar un interés por investigar la espacialización sonora, dada una peculiar ubicación de los intérpretes, tal como se muestra a continuación, con los solistas delante de los coros.

La disposición global no obedece a razones de simetría, ni de contraste, sino a la búsqueda de un juego de reverberaciones donde los ecos de los diferentes colores vocales se entrecruzan. Durante la obra, esta posibilidad es utilizada no melódicamente, sino en el plano rítmico, probablemente el más interesante en la obra. El

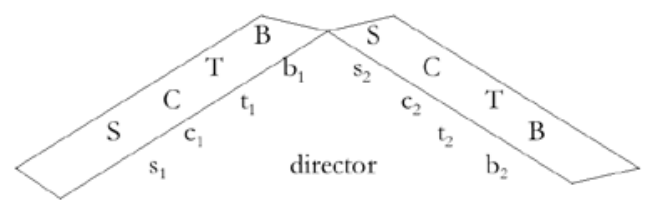

Cuadro 2: Disposición espacial de los intérpretes en De fóra (1985)

compositor parte de una secuencia básica de duraciones, de naturaleza palindrómica - ¿quizás influencia de los diseños aditivos de los ragas indios?-, expuesta en los primeros cuatro compases del bajo solista: 5-1-4-2-3-3-2-4-1-5. Cada una de las partes sigue una unidad rítmica diferente (una velocidad propia), pero todas reproducen una sucesión de duraciones derivada de la secuencia generadora:

\begin{tabular}{|c|c|c|}
\hline Voz & UNIDAD RITMICA (SEMICORCHEA) & SECUENCIA RITMIICA \\
\hline ss1 & semicorchea normal (de cuatrillo) & $2-3-3-2-4-1-5$ \\
\hline bs1 & semicorchea de septillo & $5-1-4-2 \cdot 3-3-2 \cdot 4-1-5$ \\
\hline ss2 & semicorchea de quintillo & $1-4-2-3-3-2-4-1-5$ \\
\hline b2 & semicorchea de seisillo & $5-1-4-2-2-2-4(2) 1-5$ \\
\hline sC1 & semicorchea normal (de cuatrillo) & $5-1-4-2-3-3-2-\quad-5(+7)$ \\
\hline $\mathrm{sC2}$ & semicorchea de quintillo & $1-4-2-3-3-2 \cdot 4-1-5(+7)$ \\
\hline
\end{tabular}

Cuadro 3: Relaciones entre las secuencias rítmicas del inicio (cc. 1-4) de De fóra (1985)

La soprano solista primera omite las tres primeras duraciones, la soprano solista segunda omite la primera duración, y así sucesivamente, con leves transformaciones (marcadas entre paréntesis en el cuadro). Todo este conjunto de relaciones revela la existencia de un afán poético por ordenar las duraciones, pero de tal modo que nuevamente coexisten elementos hipercontrolados (la secuencia generadora) con otros elementos, incluso en el mismo parámetro (el ritmo) aparentemente caprichosos (la elección, para cada voz, de una pauta de duraciones propia, aunque derive de la secuencia generadora, y de unas unidades rítmicas características). Desde una perspectiva exclusivamente teórica, este sometimiento a una secuencia rítmica única simultaneando distintas unidades cuenta con claros precedentes en los motetes isorrítmicos del Ars Nova. Incluso podría decirse que, hasta cierto 
punto, Macías no hace aquí sino actualizar la noción de talea, de manera que las omisiones y adiciones de valores rítmicos podrían ser interpretados como una innovación entroncada con la asimilación del serialismo postvienés, proclive a introducir transformaciones análogas en las matrices seriales.

Otro aspecto relevante de la composición, también entroncado con la tradición postweberniana, consiste en el empleo musical de las cualidades fonéticas del lenguaje, prefiriéndolas a sus denotaciones semánticas. Lo anterior se refuerza con la emisión, en algunos pasajes, con la boca cerrada, así como por la construcción de melismas articulados sobre sonidos vocálicos. Se revela así, en suma, que el tratamiento vocal propuesto se aleja de lo tradicional, favoreciendo cierto efectismo sonoro aun a riesgo de añadir dificultades de afinación y realización rítmica a los intérpretes.

Una de las composiciones más destacadas de la etapa inicial es Langsam (1985), un concertino para contrafagot con un pequeño conjunto acompañante integrado por marimba y trío de cuerdas (violín, viola y violonchelo) donde la tradición del concertare se entiende por los múltiples juegos de oposiciones establecidos entre el solista y los demás instrumentos. Los ciento diez compases de la obra mantienen de principio a fin el mismo metro (compás de $3 / 8$, a cien corcheas por minuto), pero, como es habitual en el lenguaje del autor, el tratamiento rítmico en la superficie sonora dista mucho de seguir pautas regulares. Hay fuertes contrastes de registro entre el contrafagot (que jamás supera hacia el agudo la tesitura del do\# de la octava central) y los demás instrumentos (de ámbito medio o agudo), gran variedad de técnicas de ataque y un rango dinámico igualmente amplio, mostrando el gusto de Macías por buscar la singularización de cada sonido. Este movimiento lento describe un esquema formal ternario, con una melodía prolija en ornamentación que sirve para insuflar apariencia de complejidad en la superficie sonora. Ahí detentan mayor relieve las tendencias (en cuanto a registro, dinámica, densidad rítmica o grado de saturación cromática) que la exactitud de los detalles. Se trata de una música concebida en gran medida gestualmente, en consonancia con las primeras derivaciones del serialismo integral de los primeros cursos de Darmstadt -una referencia que no puede ser casual para el compositor precisamente en estos años-.

La idea de enfrentar un instrumento con atribuciones de solista a un conjunto instrumental obtuvo con Langsam un resultado suficientemente satisfactorio como para que poco después Macías compusiese una obra de dimensiones bastante mayores: Tránsito (1987), para arpa solista, conjunto instrumental y tratamiento electroacústico en tiempo real. El ya extenso instrumentario con que cuenta la composición está agrupado de manera inusual, en cuatro grupos distintos: un trío de metales (trompa, trompeta y trombón), dos instrumentos de percusión (marimba y piano) que flanquean al arpa solista, y los cuartetos de maderas y de cuerdas. La intervención de dos transformadores y de un sintetizador de ondas como dispositivos electroacústicos completa un plantel capacitado para abarcar las más variadas gamas tímbricas.

Tránsito toma su nombre de uno de los procesos (la transición entre movimientos) que caracterizan la estructuración de la obra. Los fundamentos poéticos que la sostienen revelan un gran interés por investigar los fenómenos perceptivos en función de dos propuestas temporales (el «tiempo desplegado» y el «tiempo desplazado») que primero expone (movimientos 2 y 4) y finalmente fusiona (movimiento 6). La concepción global de la pieza se completa con un preámbulo (movimiento 1) y dos transiciones (movimientos 3 y 5) que conectan las diversas visiones temporales. Llama la atención cómo el compositor plantea el sentido orgánico de la obra, trazando en ocasiones procesos continuos de expansión interválica a partir de un centro sonoro dado, como sucede al comienzo del primer movimiento. Ahí se describe una primera ampliación por tonos enteros y de una posterior conquista de los intersticios cromáticos, en cada una de las dos fases del arranque:

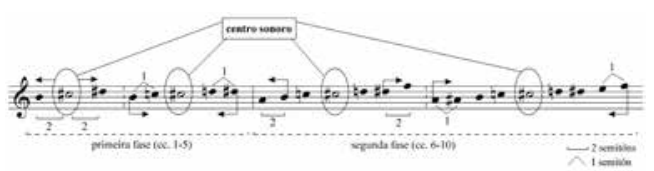

Ejemplo 5: Esquematización del proceso de expansión inicial de «l.- Preámbulo», en Tránsito (1987) 
El mismo tipo de proceso va a ocupar buena parte de la «Transición II», con un sentido de algún modo «recapitulador» que debe relacionarse con el que también se halla después, a través de la fusión de los tiempos «desplegado» y «desplazado», ya en el último movimiento. La representación musical de los dos tipos de temporalidad es el aspecto más singular de la composición. El «Tiempo desplegado», como en el segundo movimiento, se proyecta mediante la discontinuidad (cambios de compás, cambios en las pulsaciones metronómicas e inserción de pasajes medidos en segundos).

Mientras, el «tiempo desplazado» del quinto movimiento se basa en unos procedimientos más simples, derivados de las técnicas rítmicas de valores aditivos típicos en la música india y empleados en las corrientes musicales del siglo XX especialmente a partir de Messiaen (compás de valor añadido). Otro procedimiento consiste en repetir una misma secuencia melódica con pautas rítmicas variables.

La aproximación a una noción como el tiempo musical constituye una muestra muy clara del interés por cuestiones relacionadas con el ámbito de la percepción musical, que Macías investiga de manera ostensible en casi todas las obras posteriores a 1986. Uno de los casos más emblemáticos es la Sonata para piano (19861989), cuyos cuatro movimientos se fueron ensamblando en un largo proceso creativo, que explica, en parte, que su orden de sucesión pueda alterarse, conforme a una flexibilidad única en el catálogo de Macías: «Impromptu |» (1986), «Refrain» (1989), «Impromptu II» (1988) y «Paráfrasis».

Existen en estos cuatro números los dos principios básicos del estilo compositivo de sonata: el desarrollo tensional (sobre todo en el Impromptu I) y la restauración de la estabilidad inicial (aunque con perpetuas transformaciones, en la Paráfrasis final). Desde esta perspectiva se confiere una relevancia estructural menor a los movimientos centrales -por eso pueden intercambiarse- en los que puede rastrearse, también, el legado de modelos formales tradicionales: el Impromptu /l asume el carácter lúdico de los scherzi, mientras que la organización estrófi- ca del Refrain apela a una tipología frecuente en los movimientos lentos. Pero estos planteamientos son los únicos que aquí se relacionan con el pasado, en un discurso musical que se aleja de lo canónico.

La Sonata es una obra de gran complejidad: la escritura se desdobla en varios sistemas, no hay indicaciones de compás, son constantes los grupos rítmicos irregulares, así como los cambios metronómicos y las fluctuaciones agógicas, los saltos de registro y los contrastes dinámicos: la composición busca alcanzar una expresividad singular mediante la puesta en tensión de todos los aspectos que definen la configuración del fluir musical y condicionan la interpretación, derivándose parte de la expresividad de la dificultad técnica inherente a su realización. Incluso, cabe preguntarse hasta qué punto está justificada la minuciosidad con que se indican matices difícilmente perceptibles: la respuesta es que bajo la apariencia de máximo control, Macías logra infundir a su escritura una inusual fuerza expresiva. Y esta escritura casi gestual nos apunta, de nuevo, a la técnica de grupos en la primera música postserial de Darmstadt, donde la notación trasciende a su realización estrictamente literal para denotar, además, consecuencias expresivas implícitas. Formalmente, Macías plantea una oposición entre los conceptos de identidad y transformación, pero con unas cotas de complejidad hasta ahora inéditas en su música, ejemplarmente mostradas en el "Refrain» (un discurso circular, perpetuamente autorrecurrente) y la «Paráfrasis» (actualización de los materiales precedentes)

La técnica de transformación circular aplicada en el «Refrain» parte de una pequeña colección de motivos (díadas, numerados en el ejemplo) que sirve de modelo. Luego, sucesivamente, las mismas alturas, definidoras de la identidad, reaparecen con configuraciones renovadas en los demás parámetros (duración, dinámica, registro, ataque...). Como cada transformación se va operando sobre otra previa el resultado final es muy diferente del comienzo, aunque permanezca vinculado a él: 

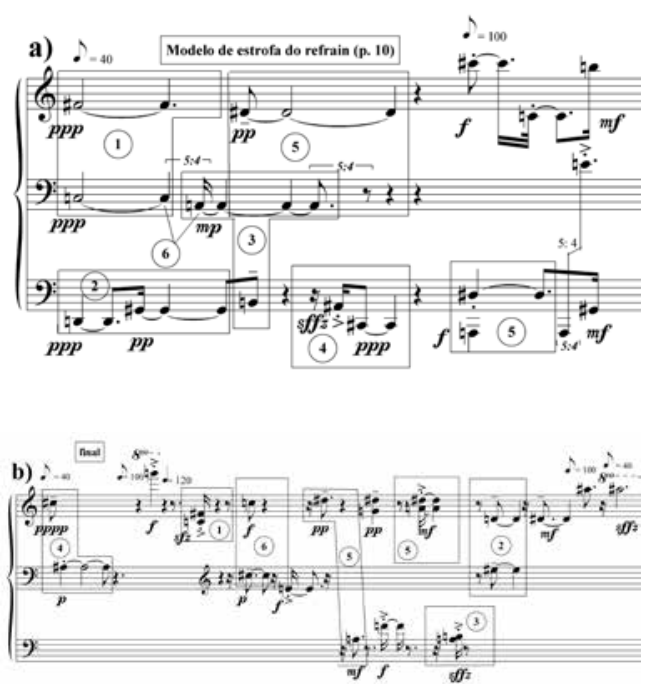

Ejemplo 6: Comparación entre: a) principio (p. 10) y b) final (pp. 17-18) de «Refrain», en Sonata (1986-1989)

Esclarecedoramente, la «Paráfrasis» titula sus tres secciones aludiendo a cómo se relacionan con los movimientos anteriores: «Destellos/ memoria (sobre "Impromptu I")», «Reflejos/ contornos/repeticiones/variaciones/fragmentaciones (sobre "Impromptu II")» y «Motivos/ giros/ frases/ incrustaciones (sobre "Refrain")».

A finales de los ochenta Macías exploró los conjuntos de cuerdas. La primera aproximación tuvo lugar con Clare I (1989), para once instrumentistas. A esta composición siguió Extracto, para cuarteto de cuerda, (1989-1991), donde la técnica de variación es imperante, trasladando las propuestas de la Sonata al ámbito cuartetístico. A mi juicio, lo más llamativo estructuralmente es la concepción y la función otorgada a la «variación», que no conlleva transformaciones en cuanto a las alturas, ni siquiera como notas de adorno: hay leves retoques en la distribución de la dinámica y en el ataque y los cambios se concentran principalmente en el ritmo, donde se operan transformaciones que hacen pensar, buscando una analogía plástica, en una deformación del tiempo musical. Esta tematización de lo temporal en música se convierte en habitual en las últimas obras de Macías y ha de relacionarse con su creciente preocupación por los procesos de percepción musical, planteados desde un enfoque estructuralista, pero sumamente flexible.

Casi a la par que la composición y revisión de Extracto, nació la que sería, junto a Duplo (1992) y Exequias (1994) una de las composiciones más amplias: Nobilissima Visione III Postludios (1989-1991), para piano, conjunto instrumental, cinta magnética y transformaciones electroacústicas en tiempo real. El juego de sucesivas «actualizaciones» de los materiales empleados por el compositor constituye por sí mismo una de las muestras más evidentes de los planteamientos poéticos que a finales de los ochenta y principios de los noventa caracterizaron el devenir estético de Macías y que básicamente consisten en la valoración de los mecanismos perceptivos y de emplear juegos «lúdicos» con la memoria. Si la idea de «parafrasear» o "comentar» materiales conocidos ya estaba presente en otras composiciones, Nobilissima Visione /l Macías da un paso más en su manejo del collage y de la llamada intercontextualidad, ya que complica el dualismo habitualmente establecido en su música entre identidad y transformación: los desarrollos de Nobilissima Visione /l son consecuencia de unos elementos también derivados (samplers) que a su vez han sido previamente confeccionados con fragmentos muy concretos de una obra anterior (el movimiento "Nobilissima Visione», de Les Adieux). Macías no trabaja con motivos, sino que compone con fragmentos, desintegra una obra original para transformar sus componentes y construir con ellos un nuevo discurso. No existe un desarrollo lineal a partir de unos materiales claramente definidos, sino que se prolongan los procesos transformadores primigenios, distorsionándolos en una nueva actualización.

Los Postludios, finalmente, son una última dilatación de los juegos de referencias múltiples que protagonizan Nobilissima Visione // $y$, en muy buena medida, son una prolongación, con inequívocas transformaciones, del hexacordo que asume el máximo protagonismo durante la composición: $I_{6}(6-15)$, con el que se inicia y concluye Nobilissima Visione /l y con el que se inicia y concluye, también, Postludios, trazándose así un doble bucle conceptual que, de nuevo, rein- 
cide en el efecto transformador -distorsionadordel tiempo sobre la memoria.

La renovación que en lo temporal opera Macías con Nobilissima Visione III Postludios como consecuencia de la red de interconexiones entre obras y fragmentos, halla una correspondencia con la espacialización de los recursos interpretativos:

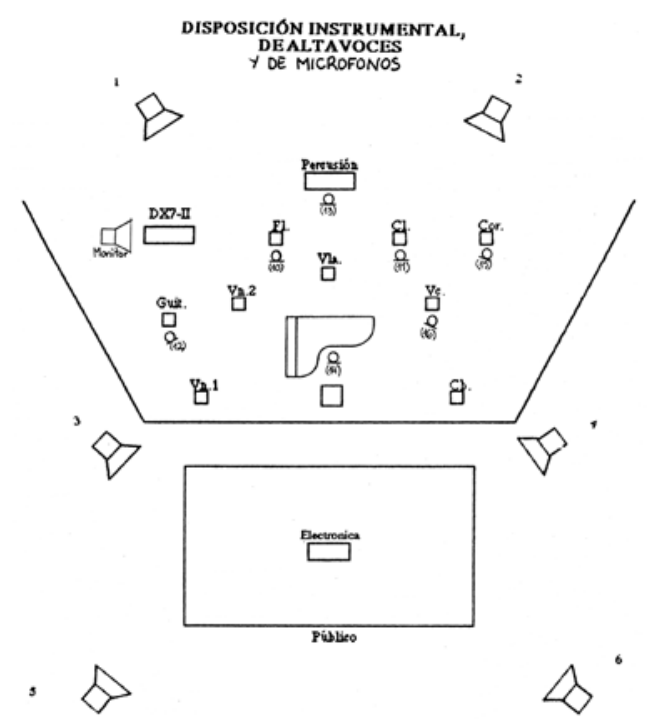

Cuadro 4: Espacialización de efectivos en Nobilissima Visione III Postludios (1988-1991)

Conforme a la disposición de los efectivos, el público recibe el sonido por una amplificación tetrafónica gracias a los cuatro altavoces que rodean el recinto reservado para los oyentes. Además, otros dos altavoces, desde el fondo del escenario, más un monitor para el sintetizador, hacen llegar al conjunto instrumental las modificaciones producidas por los dispositivos electrónicos que, mediante micrófonos, realizan tomas de sonido individualizadas a través de las cuales se habilita la realización de transformaciones en tiempo real. La colocación del conjunto instrumental en la escena no presenta ninguna particularidad reseñable, puesto que son los dispositivos electrónicos los que se encargan de distribuir el sonido en el espacio. La disposición envolvente -innovadora hace veinte años-convierte al sonido en un objeto con masa corpórea, capaz de desplazarse por el espacio. Al igual que sucedía respecto lo temporal, el tratamiento de lo espacial evidencia que la composición de la obra se gesta desde una reflexión acerca de su incidencia sobre los procesos perceptivos del oyente.

Si se trazaba un bucle semántico en cuanto a las referencias genealógicas de Nobilissima Visione III Postludios a Les Adieux, Cadencias e Interludios/ Percurso I (1989-1992) -para piano, cinta magnética y electrónica- supone un paso más en este proceso, puesto que se nutre de estas composiciones y de la Sonata para piano.

Continuando con la reutilización de materiales en Macías, Exequias (1992-1994), sobre la cual Macías llegó a proyectar una nueva «actualización» de Nobilissima Visione II, representa probablemente la culminación de esta tendencia poética. Escrita para orquesta sinfónica, sin dispositivos electrónicos, se basa en Morgengesang III (1986) y reúne varios de los tópicos en la obra del compositor: la concepción de la memoria como referente para el discurso; la reutilización con transformaciones sucesivas de los materiales conocidos, en espiral; la articulación estructural mediante paralelismos, glosas y paráfrasis; o el empleo de tácticas combinatorias y construcciones simétricas.

Utiliza Macías principios que le son propios: la «construcción» consiste en aportar nuevos materiales, la «reconstrucción» en fragmentar materiales conocidos para generar otros (como en Nobilissima Visione II), la «actualización» es una forma de variación en la que se preserva algún elemento identificable (preferentemente la sucesión de notas o el patrón básico de duraciones) y se distorsionan los demás, mientras que el «desarrollo» es la transformación más libre a partir de los materiales originales o de una parte de los mismos. Estos cuatro principios sirven para prolongar el discurso y, al mismo tiempo para articularlo. $Y$ ya no porque la aplicación de una $u$ otra técnica defina segmentos, sino, sobre todo, porque su incidencia va configurando la retórica de la pieza, el juego de tensiones basado en el conflicto determinado entre identidad y diferencia, regido por el grado de proximidad o alejamiento de los materiales básicos. Esta concepción permite ordenar el discurso de una manera 
inteligible en lo macroformal y concuerda con los juegos lógicos microestructurales (como las «actualizaciones» más básicas, sin alteraciones en cuanto a las alturas, las sucesiones rítmicas palindrómicas, o las permutaciones de elementos). El problema es que, al no resultar evidentes en muchos casos cuáles son los procedimientos transformativos empleados $y$, sobre todo, por qué se prefiere un plan «retórico» frente a otro, aunque la macroforma sea comprensible y definible, se vuelve arbitraria.

El lenguaje de Macías funciona mejor aplicado en entornos camerísticos, donde resulta más fácil equilibrar las masas sonoras y controlar en todo momento el resultado global. Durante sus últimos años, las obras camerísticas más tardías testimonian en este sentido la madurez del compositor más claramente que las obras para conjuntos mayores y, sobre todo, que las orquestales.

La chambre dans l'espace (1992-1993) está escrita para flauta, viola y guitarra. El timbre, tratado con gran equilibrio, condiciona la planificación de la dinámica, caracterizada por una moderación que no siempre se halla presente en la música del autor, y, desde luego, determina el diseño de los ataques. Frente a la fragmentación predominante en las obras de los ochenta, un aspecto que distingue de manera sustancial a esta pieza de aquéllas es el tratamiento del timbre, buscando frecuentemente fusiones de los tres instrumentos en un único gesto, un único sonido. Además, en la articulación estructural se revela con mayor claridad la red de filiaciones motívicas en la sucesión de transformaciones. En la segunda mitad de la obra, por ejemplo, destaca el uso de una secuencia melódica, que aparece originalmente en los compases 125-133 y que reaparece en varias ocasiones sometida a los tratamientos transformativos típicos en la música de Macías. A continuación presento, con fines comparativos, tres pasajes significativos ( $A, B$ y $C$ ): el inicio de los tres segmentos estructurales inmediatamente posteriores al segmento correspondiente a la secuencia melódica original. Los parecidos existentes entre las diferentes versiones, especialmente atendiendo a lo melódico, resultan más que evidentes:

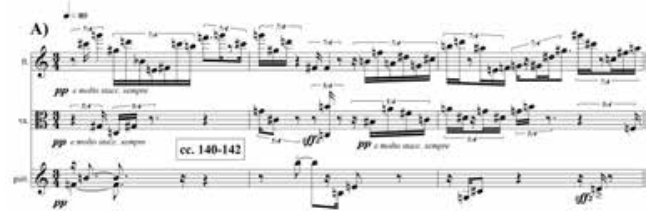

A) cc. 140-142: Variación rítmica

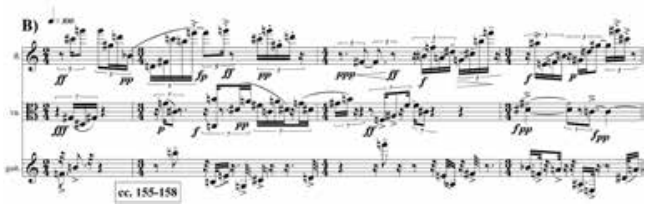

B) cc. 155-158: Variación rítmica

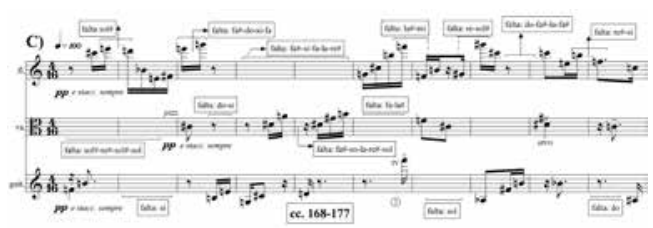

C) 168-177: Variación rítmica y elisiones melódicas

Ejemplo 7: Actualizaciones de la secuencia melódica X en La chambre dans l'espace (1992-1993)

Mientras los segmentos A y B consisten básicamente en transformaciones rítmicas, el segmento $C$ aporta otras novedades. Frente a la abundancia de tritonos en la secuencia melódica original, evidente, por ejemplo, en la parte de flauta, el segmento $C$ sustrae de la secuencia alguna de las notas, de tal manera que la incidencia de tritonos disminuye y se induce una mutación en cuanto al contenido interválico. El proceso de «fragmentación» o «disolución» melódica fruto de estas elisiones se reproduce más tarde y termina desembocando en el enunciado de una nueva propuesta melódica, con características interválicas diferentes a la secuencia $X$. El resumen de todo el proceso podría explicarse, entonces, y siguiendo los términos con los que trabajaba Macías, como: construcción (el segmento $X)$, actualizaciones (A y $B$ ), reconstrucciones (C y ss.) y nueva construcción. El conjunto traza globalmente un plan discursivo lógico, progresivo e incluso orgánico, porque cada segmento guarda algún tipo de relación respecto a su inmediato predecesor.

Adhuc (1992-1993) comparte con La chambre dans l'espace no sólo el periodo de com- 
posición, sino también los procedimientos empleados en la articulación de su estructura y, tal como he podido descubrir con el análisis, una parte de los materiales melódicos empleados. La obra cuenta con una plantilla de mayores dimensiones (que exige clarinete, trompa, vibráfono, sintetizador, violín y violonchelo) y tiene también una extensión algo superior, pero, a mi juicio, el tratamiento constructivo se encuentra más descuidado que en el trío. Baso mi valoración en el elevado grado de recurrencias existente entre los materiales empleados, bastante menos variados que en otras obras. Aunque Macías identifica en la partitura diecisiete secciones (denominadas alfabéticamente de $\mathrm{A}$ a $\mathrm{L}$ ), algunas de ellas no son sino meras repeticiones literales de otras (tal es el caso de la sección $E$, una reproducción de la sección $A$ ); un retorno a materiales iniciales que nada tiene de «actualización» y que incluso contradice la planificación estructural, basada en las transformaciones, habitual en Macías. La derivación a partir de fragmentos de La chambre dans l'espace es obvia, sobre todo en pasajes donde las referencias al trío sólo pueden calificarse como directas. El aspecto más logrado en la obra es el cuidado por las fusiones y los encadenamientos tímbricos. Lo novedoso aquí consiste en el modo de enunciación tímbrico de la idea musical objeto de la actualización: un reparto entre varios instrumentos distintos, que duplican algunas notas para reforzarlas y dotarlas de un nuevo timbre que ya no es único y fragmentario, sino que -quizás por influencia de sus experiencias en el ámbito de la electrónicase concibe de una manera sintética, mediante la fusión de los timbres individuales para crear nuevas sonoridades.

En las composiciones más tardías, el interés por el timbre se acentúa, y prueba de esto es la homogeneización tímbrica de las plantillas empleadas. Un modelo de este tipo de propuesta es el trío para violín, viola y violonchelo lubilæum (1993). Se halla estructurada en tres movimientos (I.- «De fóra», II.- «Gaudium in universo mundo» y III.- «Memorial»), relacionados entre sí mediante derivaciones motívicas. Dos aspectos merecen ser destacados de esta composición: el tipo de ilación discursiva y el recurso a sonoridades estáticas, con texturas próximas en ocasiones, incluso, a lo minimalista.
En cuanto a la propuesta discursiva, lubilæum regresa parcialmente a planteamientos de principios de los ochenta, cuando Macías construía combinando sucesivamente de distintas formas un número reducido de elementos. Pero ahora somete ese principio formalista a las vicisitudes de sus técnicas transformativas y, al mismo tiempo, utiliza la saturación sonora de una misma armonía.

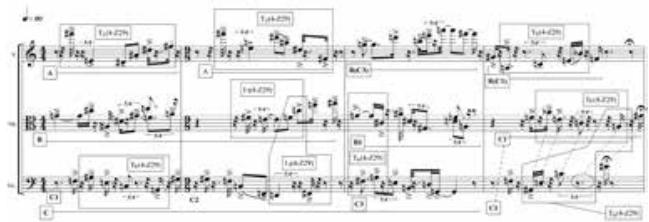

Ejemplo 8: Análisis armónico de la sección 1 (cc. 1-4) de «I.- De fóra», en lubilæum (1993)

Como muestro en el ejemplo 8, Macías extrapola la búsqueda de afinidad tímbrica a una análoga, si bien más sorprendente, comunión armónica. La afirmación maximalista de un tetracordo que se produce en el pasaje es analíticamente muy relevante por varias razones. Primero, porque se registran recurrencias tanto horizontalmente, en cada una de las voces, como verticalmente, entre partes diferentes. Segundo, porque con nueve apariciones en apenas cuatro compases su presencia roza lo que podría calificarse como saturación armónica. Tercero, porque el conjunto en cuestión es un tetracordo, cuya repetición difícilmente puede ser tan casual como la de un tricordo. Cuarto, no se trata de un tetracordo cualquiera, sino de uno de los dos que integran el único par de tetracordos en relación Z: 4-Z29. Además, como su par 4-Z15 -que, algo igualmente relevante: no es utilizado,- es un tetracordo omninterválico. En un contexto en el que se apunta la tendencia de Macías hacia la uniformización (de timbre, del material melódico, de la armonía), no puede omitirse la circunstancia de que el conjunto potenciado en el fragmento sea precisamente el que posee el vector interválico más equilibrado de toda la teoría atonal, es decir: el único capaz de tender hacia una máxima homogenización interválica. Quinto, aunque 4-Z29 aparece en nueve ocasiones, sólo muestra cuatro de sus veinticuatro formas: además de repetirse un mismo conjunto, se repiten las formas que de él se emplean. Sexto, 
porque se ponen al descubierto, por ese motivo, relaciones muy particulares: la equivalencia por transposición de tritono -entre $T_{3}(4-Z 29)$ de los primeros compases del violín y $\mathrm{T}_{9}(4-\mathrm{Z} 29)$ de las tres partes en el último compás- y la retrogradación, con un valor estructural -que empareja las apariciones de $T_{9}(4-Z 29)$ en el primer compás del violonchelo y cuarto del violín, por un lado, $y$, por otro, en el cuarto compás de la viola y el violonchelo-. Y séptimo, porque la sección está diseñada de tal modo que las tres voces concluyen, en el cuarto compás, ejecutando la misma forma del tetracordo. Lo mejor es que todas estas repeticiones, de incuestionable valor estructural, quedan diluidas en una superficie sonora marcadamente activa y dinámica, con numerosos accidentes que distorsionan la fijación subyacente. Con ejemplos como éste, donde todos los recursos empleados apuntan hacia una misma dirección (lo homogéneo), resulta muy difícil no reconocer la habilidad del compositor en aplicarse al máximo para obtener sus fines.

Pero todavía hay casos en los que el estatismo no sólo reposa bajo la superficie musical, sino que aflora hasta ella con toda la fuerza de la evidencia. Tal sucede en la compleja Alias (1993-1994), compuesta para una plantilla exclusivamente integrada por instrumentos de cuerda que, como en otros casos, deben disponerse en el escenario de manera que, por una peculiar espacialización -que recuerda a la vocal De fóra-, se potencian los efectos de reverberación y de eco.

Orgánico: II cuendas solistas (3.3.2.2.1).

Disposición instrumental:

cb.

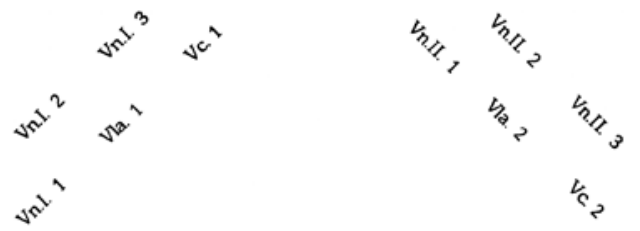

Cuadro 5: Espacialización de efectivos en Alias (1993-1994)

El aspecto más relevante de Alias es, en mi opinión, su apuesta decida por explorar el espacio musical. La distribución de los efectivos instrumentales, tal como la muestro en el cuadro, consiste en el enfrentamiento de dos quintetos en torno a un eje señalado por el contrabajo. La disposición instrumental entre ambos quintetos no responde a criterios de simetría, sino que el segundo reproduce la del primero con una rotación aproximada de unos noventa grados, algo muy similar a lo que sucedía en la coral De fóra. Al igual que allí, el resultado de este reparto, muy simple en su planteamiento, adquiere una muy notable capacidad para distribuir espacialmente el sonido, puesto que los instrumentos que se encuentran dispuestos al frente del escenario en un quinteto se hallan situados al fondo en el otro, y viceversa. De esta manera, cada instrumento cuenta con un equivalente que ocupa una posición espacial contraria y opuesta en el otro conjunto. Aunque Macías había probado en otras ocasiones a jugar con este factor espacial, ésta es la composición acústica con mejores resultados prácticos, a lo que contribuye, en no poca medida, la homogeneidad tímbrica.

La propuesta estructural de la obra se entronca con las inquietudes demostradas por Macías en composiciones previas: la memoria, la percepción musical y los juegos de dilatación y contracción temporal. Aunque el discurso es continuo, se marcan tres grandes secciones principales ( $A, B$ y $C$ ) bastante relacionadas entre sí. Las tres comienzan enunciando un mismo pasaje, que se presenta íntegro (compuesto por las once partes) al inicio de la composición $y$ que en $B$ y en $C$ se recuerda fragmentariamente (silenciando alguna de esas once partes instrumentales). Esta idea principal es múltiple, puesto que se integra como un agregado de once partes instrumentales diferentes. Existe una melodía global y ésta no es enunciada por una voz en particular, sino que es producto de la interacción de todas las partes. Pero al mismo tiempo la idea principal constituye una unidad, por cuanto sirve como referente inconfundible a lo largo de la obra. La identidad que persiste en la idea principal consiste en un proceso de exploración del espacio armónico equiparable al de Souvenir $n^{\circ} 1$ (1981-1982) y que cuenta con la nota sol como eje inicial del que brota el discurso. En cada reaparición, en las secciones, se muestra disimulada bajo otra apariencia, mas esto no es sino un subterfugio para mostrarse de otro modo. Alias representa diversas 
facetas de una misma idea, muestra diferentes posibilidades de desarrollo de la misma fuente original -sucesivas en música, pero simultáneas como potencial de actualización-. La determinación de once partes con el mismo color tímbrico presta un gran servicio a la espacialización del sonido: la homogeneidad limita las posibilidades de choques de resonancias indeseados y facilita la obtención de unos efectos de reverberación y de eco especialmente puros. El compositor explota en abundantes pasajes estas posibilidades acústicas, cuya realización se ve reforzada, por ejemplo, mediante el uso de diseños melódicos repetitivos. Para crear la sensación de un dinamismo sonoro superficial, el compositor utiliza superficies móviles, soportadas por la asignación de unidades rítmicas distintas a cada una de las seis partes instrumentales.

Una vez analizados, desde una perspectiva global, los rasgos esenciales de la música de este compositor, se hace necesario proponer unas valoraciones finales, conclusivas sobre sus posicionamientos de naturaleza estética.

\section{Conclusiones: un tiempo vivido musical- mente}

No caben dudas acerca de la apuesta de Macías por una atonalidad con claros afanes estructuralistas, pero no es tan evidente si sus planes resultan igual de lógicos en la estructura profunda como en la superficie sonora, donde es posible hallar ejemplos espectacularmente llamativos por la insistencia con la que en ellos se aplica alguna técnica de ordenamiento (permutación de elementos, fijación y saturación de armonías, empleo de patrones básicos de duraciones, etc.).

Macías busca una expresión abrumadoramente estructurada en la organización de los detalles del discurso -por supuesto, reservando siempre un mínimo margen de libertad-, lo que le confiere una apariencia de máximo intelectualismo y de suma complejidad. Pero incorpora un ingrediente de flexibilidad en la planificación macroestructural. Pone un especial empeño en someter a procesos previamente calculados los diseños superficiales (duraciones, alturas, registros, tratamiento dinámico), pero ese control es una herramienta utilizada al servicio de otra finalidad: la articulación macroestructural, que responde a principios retóricos más desarraigados de lo matemático: las ideas de tensión y estabilidad comprendidas como un conflicto entre la identidad y la alteridad. La plasmación de aspecto, y no el formalismo que impregna los rasgos más evidentes de su lenguaje musical, es el auténtico protagonista en la poética de este compositor.

Es su planificación retórica la que justifica los temas más interesantes que aparecen en su música: el transcurso del tiempo, la vivencia de la temporalidad a partir de una música que obliga a percibirla de una manera determinada, la incidencia de la memoria en la percepción musical, etc. De ahí es de donde brotan, en último término, las concepciones más originales, como las que configuran Sonata, Nobilissima Visione II/ Postludios, Exequias o lubilæum. Desde ahí se justifican los procedimientos transformativos que emplea, donde siempre subyace el conflicto en torno a la preservación y la transformación de la identidad (construcción, actualización, desarrollo, fragmentación, reconstrucción).

Así, la música de Macías transcurre en un diálogo perpetuo con el recuerdo. Pero la transformación continua a que es sometido el discurso produce un principio tensional fundamentado en el conflicto entre identidad y diferencia, entre lo recordado por la memoria y la actualización de ese recuerdo, en la que éste se muestra renovado. Este doble juego temporal y perceptivo hunde sus raíces en la aparente paradoja que comporta la «definición de lo múltiple en la voluntaria fijación de lo único», donde subyace, en último término, el impulso primigenio del desarrollo, una noción ya antigua en el bagaje de la música occidental.

Igualmente interesante es la circunstancia de que en la música de Macías sea posible hallar gestos tomados en préstamo de algunos maestros estructuralistas y, a la vez, encontrar una libertad que es reivindicada precisamente frente a los dogmatismos estructuralistas. En este dualismo radica la singularidad de la obra de Macías: su fusión entre elementos de control y elementos flexibles

Las reflexiones anteriores exigen el contraste con declaraciones de Macías, las cuales tomo de la entrevista realizada por García Alcalde: ${ }^{19}$ 
[...] Prefiro não me posicionar nem me etiquetar, que é a função dos críticos, e digo-o sem acrimónia ou ironia. [...] Mesmo un processo estruturalista [...] pressupõe surpresas para o propio autor. Como explicá-lo? Talvez analisando a partitura em detalhe, ainda que duvide que esta tarefa seja interessante para um auditório em geral. Com referências claras que não nego, creio estar a solidificar um determinado perfil [...].

$[/ \ldots]$ Penso que a estrutura não justifica nada por si mesma, porque há conceitos que surtem de maneira não premeditada e se introduzem nela. [...] Tudo parece disposto em perspectiva lógica mas, de súbito, sem se saber porquê, salta a surpresa. Quando a estrutura rege a construção da obra, esse golpe é a resposta a uma necessidade de modificar a ordem estrutural. Portanto, a estrutura é flexível, maleável e permeável a um pensamento inventivo que, por outro lado se sujeita a ela, como um fluxo que vai e vem e como o qual se xoga. Agora, para mim é fundamental conceber na origem um suporte formal.

[/...] Procuro que tudo seja consciência, mas insisto nesses elementos imprevisíveis, e de cuja justificacão na obra depende o seu nível de consequência. [...] Isso que aparece e modifica o anterior deve darlhe uma perspectiva e projectar-se sobre o succesivo. Não incluo tal coisa simplesmente porque quero, não; o processo deve ser lógico.

Las ideas principales expuestas aquí resultan bastante claras. En primer lugar, trata de desmarcarse de cualquier tendencia concreta -tarea que deja «sin acritud ni ironía» a los críticos-, en una aparente búsqueda de independencia tan habitual entre muchos creadores actuales y que puede ser interpretada como una muestra de individualismo en igual medida que como un síntoma de ambigüedad e indefinición. De ahí podría brotar esa contradicción entre la estructuración del discurso y la irrupción de elementos sorpresivos incluso para el autor. Por un lado, parece plausible pensar que un compositor pueda poner en marcha un proceso generativo cuyos resultados sonoros desconozca exactamente, o, incluso, cuyas propiedades estructurales no averigüe en su totalidad antes de iniciar la escritura de la obra.

La solución de Macías de partir de una premisa desencadenante que, en su desarrollo estructural, puede dar lugar a pasajes sujetos sólo explicables a través de lo aleatorio no hace sino demostrar un posicionamiento técnico y estético inequívocamente derivado del postserialismo y de experiencias de autores como Boulez o Stockhausen que, desde finales de los años cincuenta y durante los sesenta, lanzaron propuestas de este tipo. Pasando al ámbito español, se encuentran planteamientos bastante similares en los conceptos de «módulo» y «formante» de Luis de Pablo y Cristóbal Halffter, respectivamente. Se trata de una síntesis entre la lógica de un proceso global o de unos materiales básicos ordenados y la imprevisibilidad o la libertad que pueden caracterizar a ciertos sucesos particulares en secciones concretas de la obra o la manera de distribuir los materiales primigenios.

Por último, las especulaciones y consecuencias prácticas en Macías de la reflexión crítica sobre las aplicaciones musicales de los conceptos de memoria, cognición y percepción, así como su tematización del tiempo y el espacio o su afición al collage y la intertextualidad, hacen de su música un repertorio pionero en las investigaciones de tales cuestiones en España en general y en Galicia en particular, erigiéndose como un exponente destacado del postmodernismo musical en nuestro país, y, volviendo a la sintonía con Bal y Gay apuntada por Villanueva, "con una concepción de Galicia más grande y universal que el espacio exiguo que tenían para construir su sueño". ${ }^{20}$

\section{NOTAS}

* La realización de este artículo se inscribe entre las actividades emprendidas en el marco de mi participación en el Proyecto de Investigación Música, ideología y politica en la cultura artística durante el franquismo (1938-1975), referencia: HAR2010-17968 (Ministe- rio de Ciencia e Innovación, Proyectos Nacionales I+D/I+D+l). Deseo expresar mi agradecimiento a Miso Music de Portugal por permitirme utilizar los ejemplos musicales que ilustran el texto. La edición de todos, incluyendo su notación analítica, es mía.

1 Para consultar referencias de carácter específico sobre la música con- temporánea en Galicia, cfr.: L. López Cobas, "O terceiro terzo de século: entre a creación plural e as novas relecturas do pasado", en Historia da Música en Galicia, Ouvirmos, Sarria (Lugo), 2013, pp. 362-375; C. Villar-Taboada, "A música contemporánea en Galicia", en Gran Enciclopedia Galega. Apéndice 2005-2010. Tomo 44 (M R. Larrosa 
San Luis, dir.), Novos Vieiros, A Coruña, 2011, pp. 180-223; y "A creación musical actual en Galicia: diversidade e calidade", en Conservatorio Profesional de Música de Ourense 1957-2007 cincuenta anos de música (J.E. González Miguens, coord.), Xunta de Galicia, Consellería de Cultura, Ourense, 2008, pp. 24-34.

2 Los cinco discos compactos monográficos publicados son los siguientes: Enrique X. Macías: Nobilissima Visione II/ Postludios. Cadências e Interlúdios/ Percurso I, Miso Records, Lisboa, 1993, MCD 5 [Incluye: Nobilissima Visione II/ Postludios y Cadencias e Interludios/ Percurso l; participa Macías al cargo de la electrónica]; Santiago, Camiño de Europa: culto e cultura na peregrinación a Compostela, Miso Records, Lisboa, 1993, MCD 006 [Incluye: lubilaum, La chambre dans l'espace y Sonata; Enrique X. Macias: Duplo. Exequias (Orquestra Sinfónica de Galicia, Mark Foster), Miso Records, Lisboa, 1994, MCD 007 [Incluye: Duplo y Exequias]; y Enrique X. Macías: Itinerario de luz [Doble CD], Miso Records, Lisboa, 2008, MCD 019/020.8 [Incluye $C D$ 1: Itinerario de luz, Antistrofas, Adhuc y La lyre du désert; y CD 2 (reedición de MCD 006): lubilaeum, La chambre dans l'espace y Sonata].

Las grabaciones en discos de vinilo e incluso en cinta son muy diversas en cuanto a calidad y contenido, pero poseen el interés de presentar, en varios casos, piezas que finalmente el compositor decidió descatalogar.

3 "Macías, Enrique X. (19581995)" en Centro de Investigação e Informação da Música Portuguesa (M. Azguime, dir.), Miso Music, Lisboa [última consulta: 20/12/2013].

http://www.mic.pt/cimcp/port/ apresentacao.html?/cimcp/dispatche $r$ ? where $=0$ \& what $=2$ \& show $=0$ \&pess oa_id=3448\&lang=PT

${ }^{4}$ G. García Alcalde y E. X. Macías "Perfís de um compositor", en Enrique $X$. Macías. Nobilissima Visione III Postludios. Cadencias e Interludios/ Percurso / [librillo del CD], Miso Records,
Lisboa, 1993, MCD 005, pp. 1-5 [entrevista con Enrique $X$. Macías, publicada originalmente en castellano en el diario Faro de Vigo, el 1 de diciembre de 1991].

${ }^{5}$ R. Soutelo "Habemus Dadam" y "Quadrado de Pi", en: O Bardo na Brêtema. Artigos publicados em A Nossa Terra. 2004-2005, Arte Tripharia, Tui (Pontevedra), 2005, colección "Corpus Musicum Gallæciæ", AT-CMG-X02, pp. 64-65 y 66-67 [artículos originariamnete publicados en la sección "O Bardo na Brêtema" de A Nosa Terra, n 1173 (28/IV/2005) y no 1174 (5/N/2005)]. Se ha presentado un primer estudio sobre la actividad creativa de Soutelo: $\mathrm{C}$. Villar-Taboada, "Lo literario, lo crítico y lo lúdico: estrategias compositivas e implicaciones semióticas en Brêtema de Dom Quixote (2005), de Soutelo", en Visiones del Quijote en la música del siglo XX (B. Lolo, ed.), Centro de Estudios Cervantinos-MICINN, Madrid, 2010, pp. 321-334,

${ }^{6}$ Cfr. X. M. Carreira y M. Balboa, 150 anos de música galega, Publicacións da Xunta de Galicia, Pontevedra, 1979, serie "Arte", n² 2, pp. 53-56.

7 T. Marco, Historia de la música española: siglo XX, Alianza, Madrid, 1989, Alianza Música, $n^{\circ}$ 6, p. 296; M. Rodeiro, "Enrique X. Macías", en X Xornadas de Música Contemporánea. $\checkmark$ Centenario da Universidade de Santiago de Compostela. "Máis alá. Panorama da música galega", Universidade de Santiago de Compostela - Xunta de Galicia, CGAC, Santiago de Compostela, 1996, pp. 81-87; C. Villanueva Abelairas "Enrique Macías: debates coa soidade", en: X Xornadas de Música Contemporánea..., pp. 15-18; y C. Villanueva Abelairas, "El encuentro epistolar de dos músicos gallegos: Jesús Bal y Gay y Enrique X. Macías. Conversaciones sobre el arte/riesgo de componer en Galicia", en Delantera de paríso: estudios en homenaje a Luis G. Iberni (C. Alonso González, C. Julia Gutiérrez y J. Suárez Pajares, coords.), ICCMU, 2008, Madrid, colección "Música Hispana. Textos. Estudios", n 11, pp. 645-668.
${ }^{8}$ F. J. Cabañas Alamán, Enrique X. Macías, SGAE, Madrid, 1995, colección "Catálogos de compositores españoles".

${ }^{9}$ F. Cabañas Alamán e I. Urbelz Aja, "Macías Alonso, Enrique Gerardo", en: Diccionario de Música de España e Hispanoamérica (E. Casares, coord.), SGAE, Madrid, 2001, vol. 7, pp. 10-11.

${ }^{10}$ Véase la nota 2.

${ }^{11}$ C. Villar-Taboada, "El estructuralismo postserial en Macías", en Las músicas contemporáneas en Galicia (1975-2000): entorno cultural y estrategias compositivas. UMI Press - ProQuest, Ann Arbor (MI). pp. 557-632.

${ }^{12}$ A. Forte, The Structure of Atonal Music, Yale University Press, New Haven-London, 1973; y The Harmonic Organization of The Rite Of Spring, Yale University Press, Yale, 1978. Para una revision historiográfica de las teorías analíticas sobre atonalidad, cfr.: C. Villar-Taboada, "De la técnica al significado: debates y modelos en torno al análisis atonal", en Música, ciencia y pensamiento en España e Iberoamérica durante el siglo XX (L. Sánchez de Andrés y A. Presas, eds.), Comunidad de Madrid - Universidad Autónoma de Madrid, colección "Música y Musicología", n 1, Madrid, 2013, [I.S.B.N.: 978-84-8344-368-2], pp. 161-205.

${ }^{13}$ X. M. Carreira, 150 anos..., p. 54; y F. J. Cabañas Alamán, Enrique $X$. Macías..., p. 26.

${ }^{14}$ T. Marco, op. cit., p. 296.

${ }^{15}$ C. Villanueva (1995). "Enrique Macías: debates coa soidade", op. cit., pp. 15 y 17.

16 C. Villanueva, "El encuentro epistolar de dos músicos gallegos...", op. cit., p. 653.

${ }^{17}$ E. X. Macías, Siete micropiezas, EMEC, Madrid, 1979.

${ }^{18}$ E. X. Macías, (1982), Foglio I, EMEC, Madrid, 1982.

${ }^{19} \mathrm{G}$. García Alcalde y E. X. Macías "Perfís...", op. cit., pp. 1-2.

${ }^{20} \mathrm{C}$. Villanueva, ibidem. 